REGARDS

SUR L'ECONOMIE ALLEMAND

BULLETIN ECONOMIQUE DU CIRAC
Regards sur l'économie allemande

Bulletin économique du CIRAC

$92 \mid 2009$

Varia

\title{
Le creux de la vague bientôt atteint ?
}

Isabelle Bourgeois

\section{CpenEdition}

Journals

Édition électronique

URL : http://journals.openedition.org/rea/3741

DOI : 10.4000/rea.3741

ISBN : 978-2-8218-0880-5

ISSN : 1965-0787

Éditeur

CIRAC

Édition imprimée

Date de publication : 1 juillet 2009

Pagination : 3-4

ISSN : 1156-8992

Référence électronique

Isabelle Bourgeois, "Le creux de la vague bientôt atteint? ", Regards sur l'économie allemande [En ligne], 92 | juillet 2009, mis en ligne le 28 août 2009, consulté le 15 septembre 2020. URL : http:// journals.openedition.org/rea/3741 


\section{Le creux de la vague bientôt atteint?}

Bien que l'économie de la République fédérale connaisse la plus sévère récession de son histoire (le PIB a chuté de 3,8\% au premier trimestre 2009 par rapport au précédent et devrait reculer de quelque $6 \%$ sur l'ensemble de l'année), les Allemands gardent le moral. Les indices ifo (industrie), ZEW (finance) et GfK (consommateurs) remontent lentement, mais continûment, depuis plusieurs mois déjà. Bien sûr, " une hirondelle ne fait pas le printemps », se plat à rappeler le quotidien Frankfurter Allgemeine Zeitung (30 juin). Mais les acteurs économiques attendent visiblement l'arme au pied que la demande mondiale reprenne.

Alors que le traditionnel moteur que sont les exportations ne tourne plus qu'à très faible régime (il a tiré le PIB de 2,2 points vers le bas au premier trimestre selon Destatis) et ralentit en retour l'investissement en biens d'équipement (-1,3 point), la récession est contenue actuellement par la seule demande intérieure. La consommation publique a été en hausse de 0,8\% par rapport au $1^{\text {er }}$ trimestre 2008 . Elle devrait s'accroître nettement au second semestre sous l'effet des programmes d'investissement dans les infrastructures, adoptés dans le cadre des «paquets conjoncturels ». La consommation privée, pour sa part, a contribué de 0,3 point à la création de richesse entre janvier et mars. Et à la surprise générale des experts, le moral des ménages a enregistré un net rebond en juin, renouant avec son niveau d'avant la crise. Ceux-ci semblent sensibles aux signaux d'un certain retour au calme sur les marchés financiers, d'une relative stabilisation du système bancaire, comme des frémissements qui s'esquissent sur le marché mondial. Ils semblent aussi avoir pris confiance dans les efforts que multiplient les acteurs politiques pour endiguer la récession, que ce soit en Allemagne ou dans le monde.

Plus déterminants encore sont deux autres facteurs. D'abord la bonne tenue du marché de l'emploi. En juin, le taux de chômage (données administratives de l'Agence fédérale pour l'emploi) était de $8,1 \%$ contre $7,5 \%$ voici un an. Le nombre de chômeurs s'établissait à 3,495 millions en données CVS, soit 31000 de plus qu'en mai. Mais il ne s'agit là que des demandeurs d'emploi inscrits dans un des guichets de l'Agence, car depuis le mois de juin, ceux dont le placement est confié à un bureau privé ne sont plus comptabilisés dans les statistiques ; leur nombre s'élevait à 19000 en juin. Le fait que l'emploi se maintienne s'explique par le fait que le recul de l'activité a jusqu'ici été amorti au plan social par la baisse du volume d'heures travaillées et de la productivité horaire, comme en atteste le franc succès des mesures de chômage partiel (voir REA 90/09) : sans elles, on dénombrerait d'ores et déjà quelque 430000 chômeurs de plus (en équivalent temps plein), calcule le ministère fédéral de l'Economie. Quant à l'évolution à venir, elle est difficile à prévoir. Combien de temps encore le chômage partiel jouera-t-il son rôle de stabilisateur ? Ensuite, les premiers contrats de chômage partiel expirant à partir de juillet, et les entreprises ayant épuisé l'éventail des possibilités pour préserver l'emploi malgré le ralentissement de la production, quelle sera l'ampleur de la vague de licenciements économiques qui s'annonce?

Ensuite, le moral des ménages repose sur un taux d'inflation en forte baisse. II est tombé à zéro au mois de mai - pour la première fois depuis 22 ans -, sous l'effet du net recul des prix pétroliers et d'un retour à la normale des prix dans l'alimentation. Dans ces conditions, les Allemands envisagent de nouveau l'achat de biens de consommation durables. Certes, le commerce de détail a vu son chiffre d'affaires baisser de 0,8\% en données CVS au premier trimestre (Destatis), mais les ménages avaient profité massivement de la « prime à la casse » (les ventes automobiles ont connu un bond de $11 \%$ ) et puisé dans leurs bas de laine. S'y ajoute une légère baisse des revenus liée au chômage partiel qui touche désormais un actif sur vingt (1,4 million de salariés en juin, selon l'Agence de Nuremberg).

Mais pour soutenir la demande, le gouvernement fédéral avait décidé, dans le cadre de son deuxième programme conjoncturel, d'abaisser provisoirement, à partir du $1^{\text {er }}$ juillet, le taux de cotisation à l'assurance maladie de 15,5\% à 14,9\%. S'y ajoute,
Un seul moteur: la demande intérieure

La stabilité de l'emploi fonde la confiance des ménages

Un pouvoir d'achat relativement préservé...

... et soutenu par le gouvernement 
L'industrie prévoit une embellie

Cap de consolidation budgétaire maintenu depuis le $1^{\mathrm{er}}$ juillet également, pour les revenus inférieurs et moyens, l'exonération fiscale des cotisations versées à l'assurance maladie légale comme à l'assurance dépendance (une mesure adoptée à la suite d'un arrêt rendu en février 2008 par le Tribunal constitutionnel fédéral) et une légère hausse du niveau des pensions du régime de retraite légale. Reste à savoir comment évolueront les prix à la consommation. Hors énergie et alimentation, le taux de l'inflation était certes tombé à 1,2\% en mai ; mais si le prix du pétrole remonte sensiblement, le pouvoir d'achat risque d'autant plus de s'en ressentir que, par ailleurs, les hausses salariales négociées antérieurement sont suspendues dans nombre d'entreprises - surtout dans la branche du travail des métaux, la plus touchée par la chute de la demande mondiale-et que les partenaires sociaux renouent avec la modération salariale, du moins dans l'industrie.

Pour ce qui est du regain de confiance dans l'industrie, il « reste tiré exclusivement par les prévisions d'avenir », souligne la Bundesbank dans son rapport mensuel de juin, car les entreprises jugent leur situation actuelle «très défavorable ». Au premier trimestre, les exportations ont chuté de 21,2 \% par rapport au précédent (Destatis), la demande des pays de l'UE ayant plus fortement baissé $(-22 \%)$ que celle des marchés tiers $(-19,6 \%)$. La balance commerciale n'en est pas moins restée excédentaire, bien que de 26,8 milliards $€$ seulement. La spécialisation de l'économie allemande dans les biens d'investissement a amplifié l'impact de la contraction de la demande mondiale sur la production qui a diminué d'un quart dans cette catégorie de biens durant les trois premiers mois de l'année ; il en va de même des biens intermédiaires. Le taux d'utilisation des capacités n'était plus que de $71 \%$ en avril (contre $87 \%$ avant la crise). Mais depuis mars, l'évolution en dents de scie des entrées en commande $(+4,4 \%$ en mai par rapport à avril dans l'industrie, par exemple) ou des exportations $(+0,3 \%)$ sont autant de signes annonciateurs d'un léger frémissement de la demande extérieure. En mai, la production industrielle s'est étonnamment reprise $(+3,7 \%$ par rapport à avril), tirée principalement par la construction automobile (en partie sous l'effet des programmes de relance adoptés par plusieurs Etats). Et la chimie, autre secteur phare de l'économie allemande, constate depuis février une légère hausse des commandes dans le secteur de la chimie de base.

La relative confiance des acteurs économiques se fonde aussi sur le fait que le gouvernement fédéral ait assoupli à titre transitoire la fiscalité des entreprises, surtout des PME (voir dans ce numéro). Plus fondamentalement, sur le fait que, bien qu'il accroisse cette année le déficit de près de 90 milliards $€$ et repousse à 2013 l'horizon de l'équilibre budgétaire, le gouvernement fédéral maintienne son cap de consolidation. Certes, le Conseil de planification des finances (Finanzplanungsrat) qui réunit les ministres fédéraux de l'Economie et des Finances, ainsi que les ministres des Finances des Länder et leurs homologues des communes, vient de décider en juillet un nouvel endettement d'un montant total de 509 milliards $€$ d'ici 2013. D'ici là, l'Allemagne ne respectera pas les critères de Maastricht. Mais parallèlement à cette mesure exceptionnelle, Bundestag et Bundesrat ont adopté respectivement en mai et en juin un amendement à la Loi fondamentale. Désormais, la Constitution fait obligation au Bund de limiter d'ici 2016 tout nouvel endettement à 0,35 \% du PIB et interdit aux Länder tout recours au nouvel endettement à partir de 2020. Néanmoins, en cas de situation extrême (catastrophe naturelle, ou crise), ces clauses sont levées, à condition que Bund et Länder s'engagent sur un plan de remboursement.

TOUTE PRÉVISION SÉRIEUSE SUR LA DURÉE DE LA RÉCESSION reste néanmoins impossible actuellement, tant les facteurs à prendre en considération dans un marché globalisé sont peu maîtrisables, et tant la donne comportementale est peu intégrable dans la modélisation économique. Les effets des mesures de politique monétaire et financière sont "difficiles à évaluer dans le contexte actuel », avoue la Bundesbank. Le fait qu'ait été opéré à l'échelle mondiale « un transfert de risque sans précédent de l'économie privée vers le secteur public » a certes contribué à stabiliser la situation, mais tout en créant une situation inédite. Quant aux marchés financiers, ils restent plus que jamais imprévisibles. Les incertitudes sont grandes. Or il n'est pas à exclure non plus, à en croire la Bundesbank, que « la considération des facteurs fondamentaux prenne une importance nouvelle ». La reprise mondiale pourrait se révéler plus franche que prévu, et alors, "les économies hautement orientées vers l'exportation de biens d'investissement pourraient s'en trouver avantagées ». 